DA 1. ${ }^{a}$ CLINICA CIRURGICA DE MULHERES DA SANTA CASA - SERVIÇO DO DR. AYRES NETO)

\title{
DIAGNOSTICO EM GINECOLOGIA
}

\author{
DR. AZAEL S. LEISTNER
}

Não traremos contribuição pessoal alguma que toque á novidade, mas comentaremos apenas dois simples casos ginecologicos por nös observados no serviço cirurgico citado, para demonstrar a inexistencia de diagnosticos faceis ou que com taes não devemos contar'se, na medida do possivel, pretendermos evitar os erros desta natureza, chocantes para o profissional e não poucas vezes de furlestas consequencias para os necessitados de saude.

\section{1. ${ }^{\mathrm{a}}$ OBSERVAC̨ÃO}

Noemia A. R. - 27 anos, preta, brasileira, casada, nulipara, procedente do interior do Estado.

Ingresso na enfermaria em 4 de Abril de 1940. Registro n. 507.

Queixa: Ha 3 das tem sentido dores fortes, em forma de colica, no baixo ventre, com irradiação á região lombo-sacra. Taes dores apareceram bruscamente e vieram acompanhadas da emissão de sangue pelos genitaes externos, em quantidade moderada, lembrando uma regra comum, que já tardava de $1 \mathrm{mez}$ e meio.

Antecedentes hereditarios: Sem interesse.

Antecedentes pessoaes: Não merecem grande credito devido ao avançado gráo de idiotia da paciente. Refere comtudo, molestias proprias da infancia, pneumonia e malaria, nunca tendo sido operada.

Regras anteriores ao seu casamento, que data de 11 mezes apenas, cada 30 , 4 dias, e, apoz este acontecimento, irregulares, pois faltaram nos ultimos 1 e meio mezes, e, nos cinco mezes precedentes foram abundantes em quantidade, de maior duração, (8 dias) e compareceram duas vezes ao mez (sic).

Consultou semanas antes uma parteira formada que the assegurou achar-se gravida de 5 e meio mezes, ao que deu credito por sentir uma bola grande e movimentos estranhos no abdomem (sic). Nega partos, abortos e molestias venereo-sifiliticas.

\section{EXAME ESPECIAL DOS APARELHOS}

Aparelho respiratorio: Inspeção, palpaçẳo e ausculta, nada revelaram de anormal.

Aparelho circulatorio: Ausencia de sopros cardiacos. Bulhas normaes. Pulso ritmico e isocrono batendo 84 vezes ao minuto. Pressão arterial ao Tycos: maxima 12; minima 7,5; diferencial 4,5. 
Aparelho digestizo: Nauseas, vomitos e apetite caprichoso.

Sistema nervoso $e$ aparelhos locomotor e urinario: Normaes.

Abdomem: Á inspeção, abaulamento do mesmo, acentuado, atingindo 2 dedos transversos. ácima da cicatriz umbelical.

A palpação atribue esse achado a um grande tumor ovoidal, maior que a cabeça de um individuo adulto, disposto com grande eixo longitudinal e grossa extremidade superior, de consistencia firme, lenhosa, de mobilidade acentuada em especial lateralmente, (revelavel isso pela presssão manual e mudanças de atitude da paciente) e de escassa sensibilidade.

Ha macissez absoluta á percussão em toda a area ocupada por esse tumor que é limitado por uma linha curva, convexa para cima e dos lados, e alem da qual existe timpanismo intestinal.

Mede o mesmo de altura a partir da sinfese pubica $24 \mathrm{cms}$.

A ausculta não revela ruidos proprios dos batimentos cardiacos fetaes ou de sua movimentação, mas simplesmente pulsação da aorta abdominal e sopro das arterias uterinas.

Para o lado dos demars órgãos dessa cavidade (figado, vias biliares extrahepaticas, baço, pancreas, etc.) nada de anormal.

Aparelho genital: A inspeção mostra os genitaes exterinos banhados em sangue que flue discretamente.

Ao toque vaginal: colo amolecido, dilatado a dois dedos, atravez do qual sentem-se massas amolecidas, friaveis, constantes de coagulos e fragmentos carnosos; grande tumor, fazendo corpo com o utero, a encher, como que amoldado, a pequena bacia e dela emergente para o abdomem, onde foi reconhecido.

Sintese semeiotica: Estamos frente a uma moça em perıdo de plena atividade sexual, casada, cujos dados anamnesticos:

5 mezes consecutivos de menorragias;

Ultimos 1 e meio mezes de amenorréa;

Nauseas, vomitos, apetite caprichoso, movimentação extranha intra-abdominal ;

Colicas no baixo ventre com perdas sanguineas;

aliados aos objetivos que revelaram a presença de um grande tumor abdominopelviano, de carateristicas já descritas, levam-nos, independentemente de exames laboratoriaes (reação de Ascheim-Zondek, etc.) a afırmar a existencia da gestação, ora interrompida, e ao diagnostico diferencial seguinte:

$\left.1 .^{\circ}\right)$ Neoplasias anexiaes ou do ligamenfo largo: Aos toques retal e vaginal o tumor encontrado faz corpo com o utero que se mostra augmentado de volume. Oș anexos não são siquer perceptiveis a esses exames.

$2^{\circ}$ ) Aborto tubario.

3. $\left.{ }^{\circ}\right)$ Ruptura tubaria: Classica a interrupção da prenhez tubaria em seus dois ou tres primeiros mezes por um destes acidentes. A expulsão de coagulos sanguineos e falsa 'caduca (signal de Mac Lead) é comum no aborto tubario (influencia hormonal gravidica sobre a mucosa uterina) comtudo a dor apresentada pela enferma não era lateralizada em forma de, colicas violentas, mas hipogastrica e de intensidade discreta.

Da ruptura tubaria não havia o drama local e geral, e os signaes de Proust e Solovigo eram negativos. O grande tumor abdominal poderia coexistir com qualquer dessas complicações.

$4^{\circ}$ ) Prenhez ectopica intra-abdominal primitiva avançada: a) não ha individualização de féto e suas diferentes partes á palpação abdominal o que é sempre possivel em taes casos, devido a ausencia do envolucro uterino proprio da gravidez orto-topica; b) não existe, ao par do grande tumor encontrado, utero de tamanho, consistencia, etc., normaes, deslocado ou não por compressão.

Das modalidades dessa gravidez ectopica a com: a) feto vivo, exclue-se pela ausencia de foco e movimentos fetaes; b) feto morto, pelos dados palpatorios e ginecologicos ora citados e negativos; c) feto calcificádo (litopedium, litokelifopedium), pela falta de tumor de consistencia ossea ou cartilaginosa - 
á palpação e aos raios $\mathrm{X}$ que não revelaram̄ massa opaca anormal intrabdominal ou pelvica.

5..) Prenhez ectopica intra-abdominal secundaria: Ausencia de quadro pregresso de "aborto tubario", cuja sintomatologia - é sempre suficiente para. permitir sua suposição, atravéz uma anamnése perfeita.

6. $\left.{ }^{\circ}\right)$ Mola hidatiforme: Menorragias e desproporção uterina relativa á amenorréa; podiam indusir á tal suspeita, mas, a parada expontanea das perdas sanguineas, a consistencia firnie, ao envez de mole do utero, e, a ausencia de elıminação dos rosarios de vesiculas hidaticas (incónfundiveis) no material expulso pelos genitaes, anularan essa possibilidade.

$\left.7 .^{\circ}\right)$ Placenta prévia: As hemorragias não cederiam expontaneamente e a gravidez aparente não proseguiria a periodo tão avançado.

Ademais, seguros já da interrupção dessa gestação, recorremos á histerometria, histeroscopia e á curagem digital que não nọs revelaram a presença de féto e placenta, mas de cavidade uterina pequena, com 12 cents. de profundidade e totalmente vasia após eliminação de discreta quantidade de coagulos e fragmentos carnosos, lembrando restos ovulares.

Recorremos então á radiografia abdomino-pelviana que, mostrando ausencia de esqueleto fetal, nos.fez banir de vez a forte propensão a admitir a existencia de:

$8^{\circ}$ ) Aborto incompléto de 7 mezes: estribado em: desvalôr relativo do dado anamnest:co, tempo de amnorréa; sinaes de gestose; altura do fundo do utero; 'ausencia de vida fetal, mas atribuivel aos trez dias de perdas sanguineas.

- Restava portanto, como unico diagnostico:

9. $\left.{ }^{\circ}\right)$ Fibroma uterino, coexistindo com aborto incompleto de 1 e meio mezes: com o qual foi a doente, após devidamente preparada, encaminhada á sala operatoria em 15 de Abril de 1940.

Anestesia: Raquiana; espaço entre $2 .^{a}$ e $3 .^{a}$ vertebras lombares.

Anestesico: Neututocaina a $0,5 \%-2 \cdot \mathrm{cc}$.

Descrição da intervençáa: Laparotomia infra-umbilical cerca de 10 cents.

Aberto o peritoneo, caiu-se sobre grande tumôr, maior que uma cabeça de adulto, apresentando grosseiramente a forma em ampulhêta, emergente da pequena bacia, fazendo corpo com o utero, bastante movel, não aderente e facilmente exteriorisavel á brecha operatoria.

Histeréctomia sub-total, baixa, o mais conservadôra possivel, anexectomia esquerda, peritonisação do assoalho pelvico e fechamento completo da parede por planos.

Post-operatorio, não acidentado. Retirada dos pontos no $6^{\circ}$ dia.

- Alta curada no 9. dia, em 24 de Abril.

A peça operatoria, encaminhada ao laboratorio, foi identificada como "fibro-mioma uterino".

\section{2. ${ }^{2}$ OBSERVAC̨ÃO}

Carmen de A. L., 47 anos, branca, casada nulipara,-procedente da capital (bairro).

Entrada no serviço a 2 de Agosto de 1940.

Registro n. 1059.

Qucira: Sofreu sempre de obstipação intestinal cronica, sendo suas evacuações duras, em pelotes e só processadas á custa de laxativos ou lavagens a que recorria cada 3 a 4 dias.

Dentro desse correr de cousas, desagraclaveis, mas toleraveis, foi subitamente, ha 24 horas, surpreendida por uma dôr forte, em forma de colica que se inciava no lado direito do abdomen, atravessava o mesmo pela sua parte baixa para subir ao lado esquerdo e terminar nas visinhanças do umbigo (sic).

Essas colicas não. eram continuas, mas espaçadas de minutos de acalmia, durante os quaes sentia-se bem; desde o seu inicio não emitiu ma1s fezes. 
nem gazes, teve algumas crises de vomitos após ingestão de liquidos e seu abdomen distendeu-se gradativamente (sic).

Antecedentes hereditarios: Sem importancia.

Antecedentes pessoaes: Molestras proprias da infancia. Não é etilista, nem tabagista. Nega molestias venereo-sifilitica e corrimentos.

Menarca aos 14 anos. Regras cada 30-3 dias, mas com aspecto de sangue aquoso (sic). Ultima regra dia 10 de Julho deste ano. Casada, nulipara.

Foi operada ha cerça de 20 anos dum tumôr abdominal, cuja séde, tamanho, etc., ignora; testemunha eșsa intervenção, certamente ginecologica, a presença de uma cicatriz operatoria, por incisão de Pffannenstiel baixa.

\section{EXAME ESPECIAL DOS APARELHOS}

A cargo dos aparelhos, respiratorio, urinario, nervoso e locomotor, nada digno de nota.

Aparelho circulatorio: Bulhas abafadas, devido obesidade.

Pulso ritmico, isocrono, a 120 por minuto. Pressão' arterial ao 'Tycos: maxima, 14 e me:o; minima, 8; diferencial, 6 e meio.

Abdomem: Abaulamento uniforme e acentuado em todas as suas porções.

Sinal de Küssmaul, negativo.

Á palpação, resistencia muscular difusa predominante no flanco e fossa iliaca direitas; dôr intensa e aguda em toda a porçã̃o infra-umbilical e discreta ao nivel do epigastrio e hipocondrios.

Timpanismo generalisado á percussão. Sinaes de Kiwul, von Wahi e Jaubert, negativos.

Para o lado do figado, vias biliares, baço, rins, etc., nada de anormal.

Aparelho genital: Nos genitaes externos, pequeno tumôr do tamanho e forma duma nóz, de consistencia mole, inserido por pediculo curto e delgado na parte alta da face interna do pequeno labio esquerdo.

Ao toque vaginal, bastante dificultado pela adiposidade e reação dạ en-. ferma; colo uterino, fechado, normal em tamanho e consistencia, olhando para a parede vaginal posterior, a que se apoia francamente; utero em antero-versoflexão moveis. de volume e consistencia normaes, não sentido atravéz o fundo de saco posterior; nada de anormal a cargo dos anexos.

Sintese semïotica: Os sintômas abdominaes: Dôr aguda, expontanea, interrompida por minutos de acalmia; parada completa da emissão de fezes e gazes; meteorismo exagerado; contratura muscular de defeza da parede; vor mitos alimentares, etc., aliados ao estado geral intoxicado, á facies de sofrimento e á taquicardia, nos induziram, ante quadro tão completo de "ab̆domen agudo" ás seguintes conjeturas:

1) Abdomen agudo "pseudo-cirurgico": Dentre suas inumeras variedades, excluimos, pelo examé meticuloso dos aparelhos circulatorio, nervoso, respiratorio, etc. que nada acusaram de anormal, as seguintes: crise de angor pectoris; insuficiencias para-tiroideana e supra-renal agudas; pneumonia, broncopneumonia, processos pleuraes, etc..

2). Ulcus gastrico ou duodenal perfurado: Afastado pela ausencia de: antepassado gastrico ou duodenal, recente ou remóto; dôr em punhalada de séde preferentemente epigastrica; vomitos incoerciveis (mais exuberantes na perfuração duodena1) ; positividade dos sinaes de Gallo e Taubenschlag e Jaubert; predominancia dos sinaes para o lado do epigastrio e hipocondrios.

3) Pancreatite, aguda hemorragica: Inexistentes: a predominancia dos sintômas para a porção vizinha á cicatriz umbelical; dôr pungente, lembrando a punhalada e de séde eprgastrica; vomitos incoerciveis; referencias anaminnesticas á colicas hepaticas e perturbăções dispepticas pregressas; ' positividade do sinal de Mayo-Robson; quadro geral grave, consequente á hemorragia interna e choque peritoneal (colapso periferico); o exame de urina, que não orien- 
támos nesse sentido, dada a desnecessidade, 'darı certamente o sinal de Wohlgemuth negativo.

4) Colica hepatica: Refutavel pela ausencia de: ponto vesicular ou arca de Chauffard, dolorosa, bem comọ pela não irradiação caracteristica da dôr expontanea para o dorso, espadua direita; sintômas gastricos comuns (hiperacidez, dispepsias, gastralgia); e sub-ictericia, ictericia franca, acolia, coluria, etc.

5) Colica renal: Ás vezes se acompanha de ileo dinamico reflexo; a séde e irradiação da dôr acusada, bem como, normalidade palpatoria, percussoria, etc. de ambos os rins, e da micção e urinas emitidas (demonstrado pelo laboratorio) afastam essa origem.

6) Torção aguda do pediculo dum kisto ovariano: Fôra a doente anteriormente operada de neoplasia seguramente genital e seria portanto menor a probabilidade de tratar-se de acidente dessa origem:

Demais, não havia zona de massicez absoluta hipogastrica, propria dos tumores pelvicos volumosos, mas sim timpanismo pronunciado em todo o abdomen, cujo exame, aliado ao ginecologico, não revelou a presença de tal.

7) Processos inflamatorios agudos dos genitaes internos: A anamnese não mostrou fonte possivel dessa infecção, como seja puerperio infectado, intervenções nesses orgãos, blenorragia, corrimentos de qualquer natureza, etc., dema1s o exame dos mesmos acusou grande sensibilidade, mas o toque nada ofereceu que permitisse pensar em salpingite, piosalpinx, ovarite, pelvi-peritonite, etc. além de que não havia hipertermia, e o processo foi de aparecimento e evolução demasiado rapidos.

8) Infarto intestinal "(trombose da mesenterica): Falam contra esta entidade :

Os periodos de acalmia das colicas (em geral inexistentes); o não aparecimento de vomitos sanguinolentos, enterorragias e tumor macisso no hipogastro (consequente á paresia intestinal e enchimento das alças pelo sangue extravasado).

9) Sigmoidite perfurada: Não ha crises diarreicas anteriores, nem dor inicial vıolenta e reação muscular predominantes para a fossa iliaca esquerda.

10) Apendicite aguda: Esse diagnostico, com que foi a paciente encaminhada á Santa Casa, não era de todo infundado, pois o exame mostrou: dor expontanea e á palpação na fossa iliaca direita (pontos de Mac Burney e Lanz) em decubito dorsal e lateral esquerdo; signaes de Blumberg e Rowsing positivos; (negativos os signaes de Chutro, Metzger e Dieulafoy); a manobra de Haussmann na palpação profunda da fossa iliaca direita exacerba a dor aí localisada; abolição bilateral dos reflexos cutaneos abdominaes; ausencia comtudo da elevação termica, da discordancia entre o púlso e a temperatura tão comum nestes casos graves de apendicite entre os quaes este seria incluido si de tal se tratasse.

11) Peritonite aguda: A levar-se em conta apenas a pujança dos sintomas abdominaes seriamos forçados a pensar em peritonite aguda de origem apendicular ou diverticular (diverticulo de Mèckel) todavia os dados geraes relativos á temperatura, pủlso, relação entre ambos, e a falta de facies peritoneal, dos soluços e vomitos incoerciveis (mesmo fecaloides) emfim da cena tetrica desta funesta complicação, nos permitiram exclui-la.

12) Ileo paralitico: Nada havia do "silencio sepulcral" proprio da paresia intestinal, e sim, ao contrario, eram patentes, hiperperistaltismo (colicas e borborismos) e anteperistaltismo (vomitos), demonstrando o conjunto a luta titanica das alças, empenhadas em vencer um obstaculo certamente existente á progressão do seu conteúdo.

13) Obstrução intestinal por fecalôma: Muito embora o antepassado de constipação intestinal cronica, expontanea ou artificialmente evacuava a doente cảda 3 a 4 dias; demais seu grau de intoxicação geral não era o intenso e proprio dos portadores da molestia de Hirschprung e o quadro abdominal manifestou-se bruscamente, em plena saude, não se revelando tambem o tumôr 
arredondado, movel, de consistencia pastosa, ẹm geral de localısação baixa e muitas vezes acessivel aos toques retal e vaginal.

14) Obstrução intestinal por novelo de vermes: Mais encontradiça na creança, é, geralmente o acidente precedidó pela eliminação expontanea ou medicamentosa de vermes (ascaris, proglotes de cestodios, etc.), por via retal ou oral.

O exame de fezes foi negativo para parasitas e seus ovos.

15) Ileo biliar: Passado recente ou remoto, mesmo ligeiro, de colicas hepaticas, negativo.

16) Volvulo intestinal: Nã̃o revelavel em qualquer das sédes de predileção (alça sigmoide, colon transverso, etc.) da tumoração timpanica (sinaes de Kiwul e von Wahl) dolorosa expontanea e palpatoriamente, depressivel e acusavel á radiografia simples do abdomen pela positividade do sinal de Hintze.

17) Invaginação intestinal: Mais propria da infancia. Não encontrámos tumor cilindrico, doloroso, dotado de massicez percussoria, e de séde de predileção na fossa iliaca direita (predominancia da invaginação da porção terminal do ileo no cecum) nem evacuações sanguinolentas ou verdadeira enterorragia.

A radiografia post-clister opaco nestes casos, frequentemente faz, por si só o dragnostico, dado sinaes por demais caracteristicos, todavia dela não lançámós mão, dada urgencia do caso.

18) Hernia estrangulada externa ou interna: A possibilidade das primeiras foi seguramente afastada pelo meticuloso exame das diferentes regiões anatomicas em que costumam se processar: aneis inguinaes externos; cruraes; umbilical; triangulo de J. L. Petit; quadrilatero de Grinfelt; linha branca; linha de Spiegel, etc.

Entre a segunda modalidade, as das fossetas duodeno-jejunaes, ileo-cecaes, intersigmoideana (rarissima) e do hiato de Winslow, são pouco provaveis por se processarem geralmente á custa das alças delgadas, do que redunda oclusão alta, de sintomas mais berrantes (vomitos incoerciveis, precocemente fecaloides, e processados imediatamente em seguida á ingestão dos alimentos; deperecimento geral mais rapido, etc.) não observados por nós; as transdiafragmaticas costumam se acompanhar de sinaes toraxicos.

19) Oclusão intestinal por brida ou aderencia inflamatoria ou post-operatoria: Possivel a origem inflamatoria, mas mais provavel a post-operatoria, embora haja decorrido 20 anos da intervenção a que se submetera a paciente (taes acidentes são possiveis dias, como mezes e anos após intervenção abdominal ou melhor pelviana).

20) Oclusão intestinal por compressão neoplasica: a) Neoplasia maligna não coexistiria cóm o estado francamente florecente de enferma; b) Neoplasia suficientemente volumosa para acarretar compressão seria revelavel ao exame, contudo a extrema sensibilidade, a reação e a adiposıdade existentes, poderiam velar a lesão.

Enfim, após essas considerações sumarias, somos forçadọ a reconhecer que não passámos, na verdade, da esfera das cogitações diagnosticas, e, como quasi sempre ocorre nesses casos de "abdomen agudo cirurgico" - (em que o cirurgião, levado pela urgencia e convicção de resolver a contento e de improviso, "post-laparatomia exploradora" a surpreza que surgir dessa "caixa de segredos" que é o abdomem, ou mesmo, apenas pelo prazer de provar tal sensação) - encaminhámos a paciente á mesa operatoria, levando a "incognita etiologica" dessa "provavel" oclusão intestinal baixa.

Anestesia: raquiana a Neututocaina - 0,5\%, 2cc. Espaço entre 2. ${ }^{\mathrm{a}}$ e $3 .^{\mathrm{a}}$ vertebras lombares. 
Durante a cêna preparatoria ao ato cirurgico a doente teve descarga brusca de fezes e gazes, acarretando acentuada diminuição da distensão abdominal que, aliada ao relachamento muscular e insensibilidade (consequentes á anestesia) nos sugerio um rapido exame de controle.

Este revelou-nos um grande tumor do tamanho duma "cabeça de adulto, arredondado, emergente da bacia, atingindo a altura da cicatriz umbelical, ligeiramente desviado para o flanco e fossa iliaca direitas, de consistencia firme, mobilidade acentuada, sendo então seguramente solido, pediculado e de origem pelvica, ou melhor uterina.

Firmado o diagnostico etiologico pre-operatorio graças a este recurso da anestesia, foi a intervenção adiada para esmerado preparo da doente e realisada 4 dias após.

Anestesia geral ao balsoformio.

Operação: Laparotomia mediana supra e infra-umbilical, cerca de 15 cents. Aberto o peritoneo, mostrou-se sob as alças intestinaes meteorisadas e apoiado á parede posterior do abdomen um grande tumor com as caracteristicas já descritas, de superficie lisa, brilhante e humida, destituido de aderencias e preso á parte media da face retal do utero (normal e situado entre o pediculo e a bexiga) por um pediculo curto ( 3 cents) e delgado ( 2 cents).

Foi resecada pequena porção de grande epiploon aderente ao peritoneo parietal.

O unico tempo operatorio delicado foi a exteriorisação do neoplasma atravéz á incisão, após o que seguiu-se ligadura, secção do pediculo e fechamento completo da parede por planos.

Post-operatorio não acidentado.

Retirada dos pontos no $6 .^{\circ}$ dia. Cicatrisação per priman.

Alta curada no $8 .^{\circ}$ dia.

O exame histo-patologico da peça mostrou tratar-se dum fibromioma uterino.

\section{COMENTARIOS}

1 - A interposição das alças intestinaes meteorisadas entre o tumor e a parede anterior do abdomen (achado operatorio) a adiposidade e a contratura muscular de defeza justificam a não individualisação de tão grande neoplasma (pesando cerca de 2 kilos) inacessivel aos toques retal e vaginal.

2 - Recorrer á radioscopia e radiografia nos processos agudos cirurgicos do abdomen, em que não haja formal contraindicação ás mesmas e seja impossivel sem tão preciosos subsidios o estabelecimento dum diagnostico perfeito (anatomico, etiologico, etc) sempre compensará o tempo dispendido e poupará possiveis dissabôres.

3 - Essa regra acertada, não falhou na $1 .^{\mathrm{a}}$ observação, èm que foi decisiva e patenteou a extensão de seu valor no $20^{\circ}$ caso em que, somente aplicando-a poderiamos ter chegado, em qualquer das eventualidades a um diagnostico pre-operatorio irrefutavel. 


\section{BIBLIOGRAFIA}

1 - Tratado elemental de Ginecologia. Ahumada, J. C.

2 - Trattato completo de Ostetricia. Dr. Bumm, Ernesto.

3 - Diseases of Women. Crossen.

4 - Operative Ginecology. Crossen and Crossen.

5 - Précis de Pathologie interne. Collet, F. J.

6 - Clinica y terapeutica quirurgicas de urgencia. Garcia, M. Corachan e Alsina, F. Doménech.

7 - Percussão, ausculta e palpação dos orgãos toraxicos e abdominaes. Dr. - Dünner, L. e Neumann, R. Tradução de H. Jobin e R. Margarido da Silva.

8 - Traité de Gínecologie medico-chirurgicale. Faure, J. L. e Siredey, A.

$9 \rightarrow$ Tratado de Ginecologie. Hofmeier, M. e Schoereder, C.

10 - Das crises dolorosas abdominaes. Prof. Kuttner, L. e Dr. Krueger, K. Isaac. Tradução do Dr. H. Jobin e Dr.'R. Margarido da Silva.

11 - Practical surgery of the abdominal and pelvic regions. Kennedy, J. William.

12 - Traité medico-chirurgical de Gynecologie. Lagrave, F. Labadie e Legueu F.

13 - Chirurgie d'urgence. Lejars, F.

14 - Peritonite perfurative. Moncálvi, L.

15 - Abdomen agudo quirurgico. Pavlovsky, J.

16 - Operative Ginecology. Peham and Amreich.

$17 \div$ Modern surgical technic. Thorek.

\section{Laboratorio de Analyses}

"ALEXANDRINO PEDROSO"'

RUA D. JOSÉ-DE BARROS, 168

$2:^{\circ}$ ANDAR - TELEPHONE: 4-5239

SÃO PAULO 